\title{
Intracranial aspergillosis mimicking a middle cranial fossa tumor involving the cavernous sinus in an immunocompetent patient: Case report and literature review
}

\author{
Mohamed Badri $^{1}$, Bouali Sofiene ${ }^{2}$, Boubaker Adnene ${ }^{2}$, Bahri Kamel ${ }^{1}$, Jemel Hafedh ${ }^{2}$, \\ Zammel Ihsen ${ }^{1}$ \\ ${ }^{1}$ Department of Neurosurgery, Burns and Trauma center, Ben Arous, Tunisia \\ ${ }^{2}$ Department of Neurosurgery, National Institute of Neurology "Mongi Ben Hmida", Tunis, Tunisia
}

Email address:

badri.med@gmail.com (M. Badri)

To cite this article:

Mohamed Badri, Bouali Sofiene, Boubaker Adnene, Bahri Kamel, Jemel Hafedh, Zammel Ihsen. Intracranial Aspergillosis Mimicking a Middle Cranial Fossa Tumor Involving the Cavernous Sinus in an Immunocompetent Patient: Case Report and Literature Review.

American Journal of Psychiatry and Neuroscience. Vol. 2, No. 4, 2014, pp. 56-59. doi: 10.11648/j.ajpn.20140204.12

\begin{abstract}
Intracranial aspergillosis is rare in immunocompetent patients. Extension to the cranial base via the sphenoid sinus is exceptional. Authors report a case of middle cranial fossa aspergillosis involving the cavernous sinus and mimicking an extra-axial tumor in an immunocompetent 65 year-old male. The patient had been suffering from left trigeminal neuralgia and a horizontal diplopia since 4 months. Physical examination featured a left complete ophtalmoplegia and an hyperesthesia of the left maxillary nerve. Radiological examination showed a left temporal extraaxial temporal expanding intracranial lesion, involving the left cavernous sinus with extension to the sphenoidal sinus. A large tumor resection was performed through a left fronto-temporal craniotomy with no postoperative complications. Pathologic examination and specimen culture concluted to an Aspergillus fumigatus. Authors discuss the clinical, radiological features and management of such lesion through a literature review.
\end{abstract}

Keywords: Aspergillosis, Middle Cranial Fossa, Cavernous Sinus, Sphenoid Sinus

\section{Introduction}

Fungal infections of the central nervous system (CNS) are life threatening diseases with no clinical or radiological characteristics. The use of steroids, granulocytopenia and immune dysfunctions like systemic autoimmune diseases and organ transplants are the major predisposing factors to the development of CNS fungal infections. Aspergillosis is an ubiquitous fungal infection which is localized most frequently in the lung ${ }^{[23]}$ and may colonize sini of the face by Aspergillus's spores inhalation. CNS aspergillosis is rare with a high mortality and most frequently occurs as an opportunistic fungal infection ${ }^{[5,12]}$. Extension into the cranial base is rare and it occurs when the sini of the face are involved.

\section{Case Presentation}

A 65-year-old male, without any previous medical records, had been suffering from left facial neuralgia since 4 months. This left trigeminal neuralgia was paroxystic without a trigger zone and never disappears between paroxysms. He was referred to our department for a recent establishment of a horizontal diplopia.

The neurological examination featured a left complete ophtalmoplegia and an hyperesthesia of the left maxillary nerve. The lid reflex was intact. Other physical findings were normal and no abnormalities were noted on blood tests.

Radiological examination including computerized tomography (CT) scan displayed a left extra-axial temporal expanding intracranial lesion, poorly-defined and isodense surrounded by brain edema. Osteolysis of the sphenoid 
sinus wall was noticed. This tumor enhanced homogenously and strongly, it appears to invade the left cavernous sinus and the optical canal and a mass effect on the left temporal horn was found (Fig. 1 A \& B).

On Magnetic resonance imaging (MRI) the tumor appeared to be tissular located in the middle cranial fossa closely to the cavernous sinus. The lesion appeared isointense on T1 weighted images (WI), heterogeneous and hyperintense with a hyposignal of the boundaries on T2-WI. The lesion enhanced markedly and heterogeneously after Gadolinium administration (Fig. 2 A \& B). Extensions were made to the sphenoidal sinus and the orbital cavity suggesting a malignant tumor.

Decision was to have a pathologic proof surgically. Through a left fronto-temporal craniotomy, alarge but not total gross resection of the tumor was performed. The invasion of the cavernous sinus has obliged us to interrupt the removal of the tumor. The postoperative course was uneventfull and the patient was discharged on the 7th postoperative day.

Pathologic study demonstrated a polymorphic inflammatory reaction made by lymphocytes, plasmocytes, macrophages and polymorponuclear neutrophils within nodular lesions. The center of these lesions was occupied by long and ramified strands with septate evoking strongly an Aspergillus infestation (Fig. 3). Aspergillus fumigatus was isolated in histological specimen culture. HIV serology was then carried on and turned out to be negative.

Anti-fungal drugs were prescribed immediately using Voriconazole for 3 months. Trigeminal neuralgia and oculomotor palsy had partially improved. 8 months later, the patient is still taking drugs and showed a fair outcome.
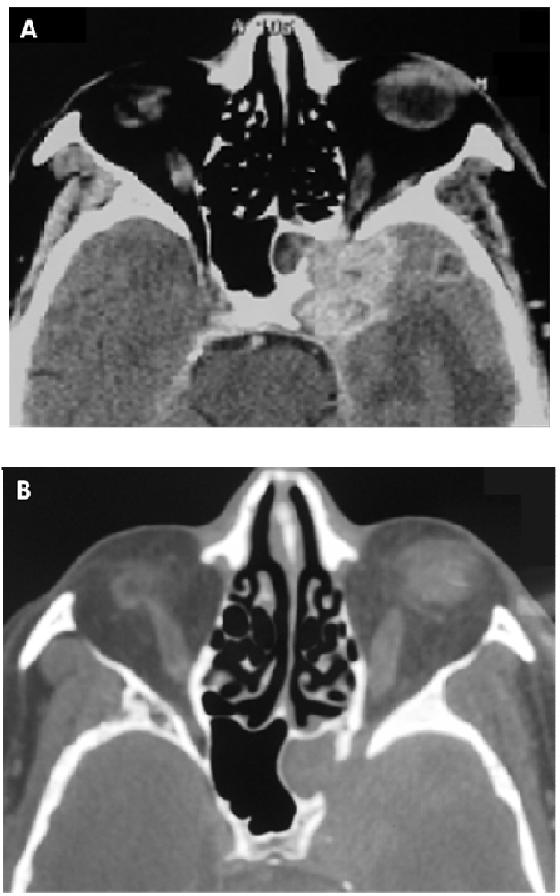

Figure 1. A, cranial axial computed tomography with contrast enhancement shows a space occupying lesion of the middle cranial fossa. $B$, bone window shows bone erosion of the sphenoid sinus wall.
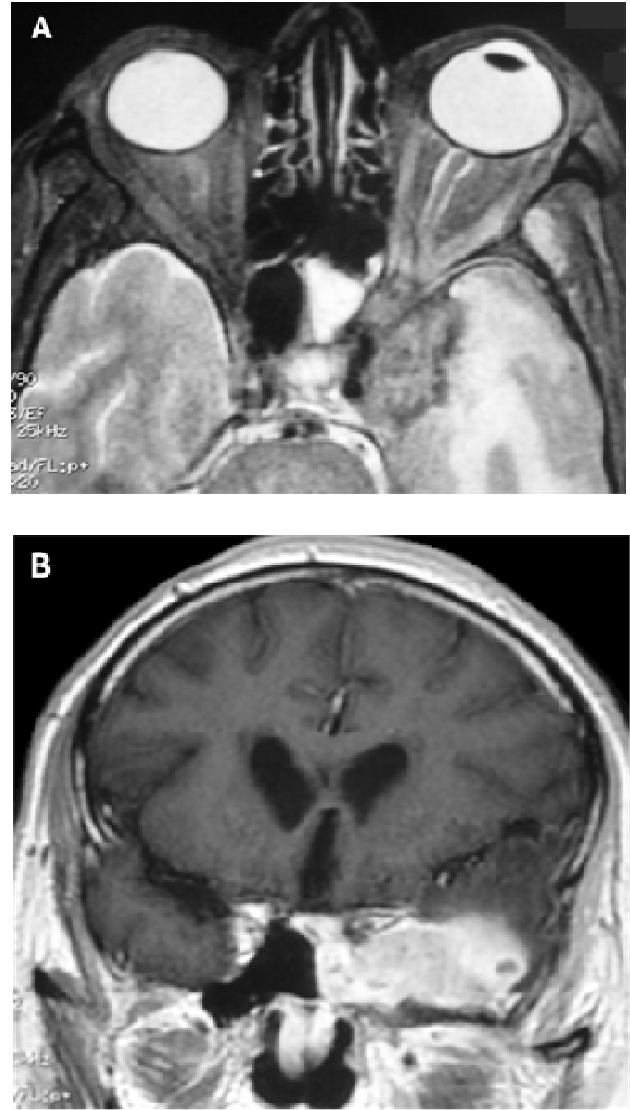

Figure 2. A, axial T2-WI displays an heterogeneous signal of this lesion. B, post-enhanced coronal T1-WI revealing an intense contrast enhancement of this intracranial mass involving the cavernous sinus.

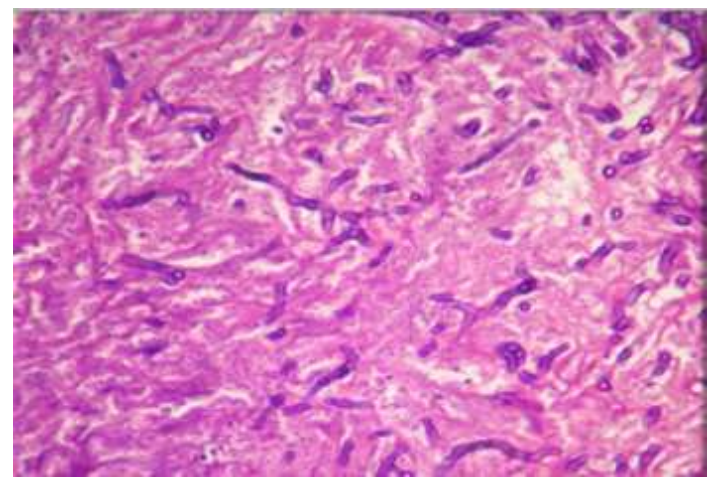

Figure 3. Photomicrograph revealing a polymorphic inflammatory reaction with septate hyphae of Aspergillus.

\section{Discussion}

Of the 350 species of Aspergillus, A. fumigatus is most frequently associated with human disease [6] and It isolated in $90 \%$ of the cases [13]. Aspergillosis is frequently implicated as a cause of invasive aspegillosis in immunocompromised patients [22]. However, it can occasionally affect immunocompetent hosts [19, 23]. In the present case, the patient is not immunocompomised and he did not have any medical history of pulmonary tuberculosis, narcotic addiction, diabetes, autoimmune disease or rheumatologic disorders that might have predisposed him 
to intracranial aspergillosis.

Hematologic dissemination and direct extension from adjacent areas such as paranasal sini or orbits are the two mechanisms through which intracranial aspergillosis may result.

Invasive aspergillosis can involve the skull base with often an extension into the orbit and cranial cavites [16, 20]. Sphenoid aspergillosis has been described as the most aggressive location because of the close relation to the skull base $[11,15,18]$ and the high risk of intracranial extension.

Aseprgillosis of the sphenoid sinus can extend into the orbit [1], the cavernous sinus, the middle cranial fossa and the temporal lobe $[7,10]$ as observed in our case. The involvement of the cavernous sinus is rare $[4,25]$. This location can lead to severe complications due to cavernous sinus thrombosis or possible cerebral infarction [4].

Symptoms and signs of intracranial aspergillosis are non specific and depend on location of the disease and type of CNS involvement $[19,21]$. Neuro-imaging in cerebral and craniofacial aspergillosis are also non specific [4]. When aspergillosis involves the skull base, it is sometimes difficult to differentiate aspergillosis abscess or granuloma from malignant neoplasm [28]. MRI may help to differentiate between paranasal inflammatory tissues and neoplasms [8]. Aspergillosis produces extremely low signals on T2-wenghted images which is explained by a strong concentration of ferro-magnetic elements such as iron, zinc and magnesium in aspergillosis mass $[9,28]$. According to Yamada et al. Gadolinum enhancement of aspergillosis is more important in immunocompetent patterns. This fact is explained by the better host-defense potential throughout a strong inflammatory reaction [27]. CT scan show often a bony erosion of the sinus walls and/or skull base [29].

A preoperative diagnosis of aspergillosis is very difficult $[2,26]$. In most cases, smears and cultures of the cerebrospinal fluid are negative and only histological examination of specimens obtained by surgery, biopsy or autopsy can confirm the diagnosis of aspergillosis.

Management of intracranial aspergillosis depends on host immunity and the degree of tissue invasion [1]. Systemic administration of antifungal agents is essential for the treatment of intracranial aspergillosis. Amphotericin B is no longer recommended because its systemic toxicity [23] and it is replaced by Voriconazole which is better tolerated for all forms of invasive aspergillosis especially in intracranial involvement [14].

Surgical resection is indicated whenever aspergillosis granuloma exert a mass effect on the brain. Debridement of the involved sinus is often necessary.

In some cases, it has been demonstrated that antifungal treatment can be effective without surgical procedures when aspergillosis involves the cavernous sinus and the skull base[3,17]. Acording to Panda et al., the combination of these antifungal drugs for skull base aspergillosis may be effective [17], with low risk. Intracranial aspergillosis has often a bad outcome with high mortality [13]. However, prognosis seems to be better in craniocerebral Aspergillosis of sinonasal [4, 24].

\section{Conclusion}

Aspergillosis of the cavernous sinus and the middle cranial fossa is rare. Imaging features are not specific and the positive diagnosis is only histological. Thus, it should be included in the differential diagnosis of lesions involving the cranial base. Severe complications may occur if it is misdiagnosed or untreated due to its progressive nature. Early diagnosis, surgical removal of involved tissues and anti-fungal drug administration are the bases of an effective therapy.

\section{References}

[1] Akhaddar A, Gazzaz M, Albouzidi A, Lmimoni B, Elmostarchid B, Boucetta M. Invasive Aspergillus terreus sinusitis with orbitocranial extension: case report Surg Neurol 2008;69:490-495.

[2] Alapatt JP, Kutty RK, Gopi PP, Challissery J. Middle and posterior fossa aspergilloma. Surg Neurol 2006;66(1):75-79.

[3] Browning AC, Sim KT, Timms JM, Vernon SA, McCanachie NS, Allibone R, et al. Successful treatment of invasive cavernous sinus aspergillosis with oral itraconazole monotherapy. J Neuro-Ophtalmol 2006;26(2):103-106.

[4] Chandra S, Goyal M, Mishra NK, Gaikward SB. Invasive aspergillosis presenting as a cavernous sinus mass in immunocompetent individuals; report of 3 cases. Neuroradiology 2000;42:108-111.

[5] Cho YS, Lee DK, Hong SD, Oh WS. Intracranial aspergillosis involving the internal auditory canal and inner ear in a immunocompetent patient. Am J Neuroradiol 2007;28(1):138-140.

[6] Dyken ME, Biller J, Yuh WTC, Fincham R, Moore SA, Justin E. Carotid-cavernous sinus thrombosis caused by aspergillus fumigatus: magnetic resonance imaging with pathologic correlation-a case report. Angiology 1990;41:652-656.

[7] Ederies A, Chen J, Aviv RI, Thompson AL, Symons SP. Aspergillosis of the Petrous Apex and Mechel's Cave. Skull Base 2010;20(3):189-192.

[8] Eskey CJ, Whitman GJ, Chew FS. Invasive aspergillosis of the orbit. AJR Am J Roentgenol 1996;167:1588.

[9] Fellows DW, King VD, Conturno RN, Bryan RN, Merz WG, Zinreich SJ. In vitro evaluation of MR hypodensity in Aspergillus colonies. Am J Neuroradiol 1994;15:1139-1144.

[10] Freudenstein D, Koerbel A, Beschorner R, Tatagiba M. Successful treatment of Aspergillus granuloma involving the cavernous sinus and the middle fossa by using surgery and viroconazole. J Neurosurg 2007;106:511.

[11] Fuchs HA, Evans RM, Greek CR. Invasive asergillosis of the sphenoid sinus manifested as a pituitary tumors. South Med J 1985;78:1635-1637. 
[12] Garcia RJ, Toyra P, Edwards C. Invasive aspergillosis with central nervous system dissemination in a presumably immunocompetent, non-neutropenic patient: case report and review. South Med J 2006;99 (6):607-610.

[13] Genzen JR, Kennedy B. Central nervous system Aspergillus infection after epidural analgesia: diagnosis, therapeutic challenges and literature review. Diag Microbiol Inf dis 2009;65:312-318.

[14] Herbrecht R, Denning DW, Patterson TF, Bennett JE, Greene RE, Oestmann JW, et al. Viroconasole versus amphoterecin B for primary therapy of invasive aspergillosis. N Engl J Med 2002;347:408-415.

[15] Kurita H, Shiokawa Y, Furuya K, Segawa H, Sano K. Parasellar Aspergillus granuloma extending frem the sphenois sinus: report of two cases. Surg Neurol 1995;44:489-494.

[16] Neilson EW, Weisman RA, Savino PJ, Schatz NJ. Aspergillosis of the sphenoid sinus presenting as orbital peudotumor. Otolaryngol Head Neck Surg 1983;91:699-703.

[17] Panda NK, Saravanan K, Chakrabati A. Combination antifungal therapy for invasive aspergillosis: can it replace high-risk surgery at the skull base. Am j Otolaryngol 2008;29:24-30.

[18] Petrick M, Honegger J, Daschner F, Feuerhake F, Zentner J. fungal granuloma of the sphenoid sinus and clivus in a patient presenting with cranial nerve III paresis: case report and review of the litterature. Neurosurgery 2003;52:955-959.

[19] Saini J, Gupta AK, Jolapara MB, Chatterjee S, Pendharkar $\mathrm{HS}$, Chandreshekher $\mathrm{K}$, et al. Imaging findings in intracranial Aspergillus infection in immunocompetent patients. World Neurosurgery 2010;74(6):661-670.

[20] Sarti EJ, Blaugrund SM, Lin PT, Camins MB. Paranasal sinus disease with intracranial extention: aspergilosis versus malignancy. Laryngoscope 1988;90:748-754.
[21] Siddiqui AA, Shah AA, Bashir SH. Craniocerebral aspergillosis of sinonasal origin in immunocompetent patients: clinical spectrum and outcomes in 25 cases. Neurosurgery 2004;55(3):602-613.

[22] Sood S, Sharma R, Gupta S, Pathak D, Rishi S. Neuroaspergillosis in an immunocompetent patient. Indian $\mathrm{J}$ Med Microbiol 2007;25(1):67-69.

[23] Srinivassan US. Intracranial aspergilloma in immunocompetent patients successfully treated with radical surgical intervention and antifungal therapy - case series Ann Acad Med Singapore 2008;37(9):783-787.

[24] Tierney P, Thomas M, Samuel D, Patel KS, Stafford N. Recurrent aspergilloma of the frontoethmoidal sinus in a non-immunocopromised patient. J R Sos Med 1996;89:165166.

[25] Urculo E, Aranzadi MJ, Ruiz I, Villanua J. aspergillus granuloma of the cavernous sinus: magnetic resonance imaging with pathologic correlation. Acta Neurochir (Wien) 2005; $147: 341-342$.

[26] Walsh TJ, Hier DB, Caplan LR. Aspergillosis of the nervous system: clinicopathological analysis of 17 patients. Ann Neurol 1985; 18:574-582.

[27] Yamada K, Shrier DA, Rubino A, Shan Y, Zoarski GH, Yoshiura $\mathrm{T}$, et al. Imaging findings in intracranial aspergillosis. Acad Radiol 2002;9:163-171.

[28] Yamada K, Zoarski GH, Rothman MI, Zagardo MT, Nishimura T, Sun CCJ. An intracranial aspergilloma wiyh low signal T2-weighted images corresponding to iron accumulation. Neuroradiology 2001;43:559-561.

[29] Zinreich SJ, Kennedy DW, Malat J, Curtin HD, Epstein JI, Huff LC, et al. Fungal sinusitis: diagnosis with CT and MRI imaging. Radiology 1998;169:439-444. 